\title{
TAXA DE POBREZA E POPULAÇÃO RURAL NO BRASIL: ANÁLISE ESPACIAL DOS PERÍODOS 2000 E 2010
}

\section{Marcio Marconato}

Doutorando do Programa de Pós-Graduação em Economia pelo PCE/UEM

Endereço para contato: Rua Ítalo Moro, 168 - Vila Estrela - Ponta Grossa - PR.

CEP: 84050-230 - E-mail: marconatoce@bol.com.br

\author{
Marcos Aurélio Brambilla \\ Mestre em Economia Regional pela Universidade Estadual de Londrina (UEL). \\ Endereço para contato: Avenida Santo Berloffa, 63 - Centro - Paiçandu - PR \\ CEP: 87140-000 - E-mail: marcos-brambilla@hotmail.com
}

\section{Marcia Regina Gabardo da Camara}

Professora Associada do Departamento de Economia da Universidade Estadual de Londrina (UEL). Endereço para contato: Rua Belo horizonte, 734 - Londrina - PR

CEP: 86020-060 - E-mail: mgabardo@uel.br

\section{Sérgio Carlos de Carvalho}

Professor da Universidade Estadual de Londrina (UEL).

Endereço para contato: Rodovia Celso Garcia - PR 445, Km 380, s/n - Campus Universitário, Londrina - PR, Departamento de Economia.

CEP: 86057-970 - E-mail: sc.carvalho@terra.com.br

\section{Sidnei Pereira do Nascimento}

Professor da Universidade Estadual de Londrina (UEL)

Endereço para contato: Rodovia Celso Garcia - PR 445, Km 380, s/n - Campus Universitário, Londrina - PR, Departamento de Economia.

CEP: 86057-970 - E-mail: sidnei@uel.br

Recebido em 19 de março de 2015. Aceito em 25 de setembro de 2015.

\section{RESUMO}

O objetivo do presente artigo é analisar a distribuição espacial da pobreza rural nos municípios brasileiros e a relação entre população rural e taxa de pobreza nos anos de 2000 e 2010. O trabalho utiliza a Análise Exploratória dos Dados Espaciais para identificar o coeficiente bivariado I de Moran, o gráfico de dispersão, o mapa de significância e finalmente o mapa de cluster das variáveis dos municípios. Os dados foram coletados no Instituto Brasileiro de Geografia e Estatística (IBGE) através dos censos de 2000 e 2010. Verificou-se que entre 2000 e 2010 ocorreu um aumento pouco expressivo da autocorrelação espacial positiva entre o percentual da população rural e taxa de pobreza para os municípios brasileiros. A conclusão do estudo é que, as cidades nas quais se concentram uma grande população rural continuam apresentando uma alta taxa de pobreza, confirmando a hipótese de que a população rural tem dificuldade em usufruir de políticas de combate à pobreza devido ao pouco acesso a essas informações e pelo distanciamento físico. Por outro lado, há indícios que o Programa Nacional de Fortalecimento da Agricultura Familiar (PRONAF) surge como uma política que pode contribuir para minimizar os seus efeitos, mesmo que lentamente reduzir a pobreza rural.

\section{Palavras-chave}

Pobreza, AEDE, Rural. 


\begin{abstract}
The purpose of this paper is to analyze the spatial distribution of rural poverty in brazilian municipalities and the relationship between rural population and poverty rate in 2000 and 2010. For this, the work utilizes the Exploratory Spatial Data Analysis to identify the bivariate moran's I coefficient, the scatter plot, the significance map and ultimately the cluster map of the municipalities variables. The data were collected in the Brazilian Institute of Geography and Statistics (IBGE) through the censuses of 2000 and 2010. It was found that between 2000 and 2010 there was a small increase in the positive spatial correlation between the percentage of rural population and poverty rate to brazilian municipalities. The conclusion of this paper is that the cities in which they concentrate a large rural population continue to show a high rate of poverty, confirming the hypothesis that the rural population has difficulty benefit from policies for combating poverty because of poor access to this information and the physical distance. On the other hand, there are indications that the National Family Farming Strengthening Program (PRONAF) emerges as a policy that can contribute to minimize its effects, even if slowly reduce rural poverty.
\end{abstract}

\title{
Keywords
}

Poverty, ESDA, Rural.

\section{Introdução}

Desde os anos 50 o Brasil vem passando por um processo contínuo de urbanização que se refletiu na queda da participação da população rural nos municípios. Em 1950 o país tinha aproximadamente 33 milhões de pessoas vivendo em áreas rurais o que representava 64\% da população total da época. Em 2010 a população rural era em torno de 29 milhões, representando $15 \%$ do total. Apesar de o percentual ter se reduzido ao longo do tempo, o número de pessoas residentes no meio rural é ainda elevado.

Nos anos 80 houve uma verdadeira urbanização de algumas áreas do meio rural brasileiro, deixando de ser apenas um espaço agropecuário (cultivos e criação de animais), ganhando importância como moradia, lazer e preservação ambiental (SILVA, 1998).

O PIB da agricultura representou em torno de $15 \%$ do Produto Interno Bruto brasileiro no ano de 2013 segundo o Centro de Estudos Avançados em Economia Aplicada (CEPEA, 2015). Segundo o Instituto Brasileiro de Geografia e Estatística (IBGE, 2015) o número de pessoal ocupado na agropecuária é de aproximadamente 14 milhões de pessoas, já o número de estabelecimentos é de 5,1 milhões de acordo com o Censo Agropecuário de 2006. No entanto, apesar das dimensões do setor, estudo realizado pelo Instituto de Pesquisa Econômica Aplicada (IPEA, 2012) mostra que a pobreza é maior nas áreas rurais do que nos centros urbanos, embora o percentual de pobres na área rural venha diminuindo.

A pobreza rural no Brasil diminuiu consideravelmente nas últimas duas décadas, mas continua a ser um problema sério e foco importante das políticas públicas. Grande parte do declínio é atribuída a inovações das políticas de seguridade social e de transferências condicionais de renda. Sem dúvidas ambas as políticas tiveram um impacto significativo sobre a pobreza em suas fases iniciais. O Plano Real, lançado em 1994 foi outro fator importante para a redução da pobreza (HELFAND; PEREIRA, 2012). 
A pobreza rural deve ser estudada e tratada de forma diferenciada, porque ela apresenta várias especificidades em relação à pobreza urbana. De acordo com Mocelin (2010), a pobreza rural tem condicionantes específicos: forma do acesso a terra, dificuldade de acessar serviços sociais básicos e baixo nível de escolaridade das pessoas. Os pobres urbanos, em certa medida, têm proximidade com as organizações (governamentais e não governamentais) que realizam ações de combate à pobreza, facilitando relativamente o acesso às políticas públicas. Já para as comunidades pobres rurais este acesso é dificultado, principalmente, pela falta de informação, distanciamento físico e a não assistência das organizações de extensão rural (MOCELIN, 2010).

Contudo, apesar das adversidades, Kageyama (2003) destaca a crescente diversidade de rendas dos domicílios agrícolas, como um fator importante para a superação da pobreza rural. Mattei (2001, 2005), Sartor et al. (2014) e Batista e Neder (2014) apontam o Programa Nacional de Fortalecimento da Agricultura Familiar (PRONAF), como uma importante política pública para o combate à pobreza rural, mas que sua a ação é indireta e depende das especificidades das regiões analisadas.

Neste contexto, o objetivo do presente artigo é verificar se existe relação espacial entre o percentual da população rural e taxa de pobreza nos municípios brasileiros nos anos de 2000 e 2010. Conforme a ideia de Mocelin (2010, 2011), adota-se a hipótese de que a população rural tem dificuldade em usufruir de políticas de combate à pobreza devido à dificuldade do acesso a essas informações e pelo distanciamento físico. $\mathrm{O}$ artigo está dividido em cinco partes, incluindo a introdução. Na segunda parte do estudo discute-se o referencial teórico sobre o tema. Na terceira parte apresenta-se a metodologia usada na pesquisa. No quarto item são discutidos os resultados e por último apresentam-se as conclusões.

\section{Referencial teórico e empírico sobre pobreza}

Amartya Sen (2001) destaca que a pobreza é não somente uma inadequação da renda, mas também envolve privações de capacidades fundamentais que não estão inseridos no seu conjunto orçamentário e outras formas de riqueza ou renda, podendo envolver características pessoais e características externas. Logo, diferentes pessoas podem apresentar diferentes necessidades, as quais envolvem dimensões econômicas e não-econômicas (SANTOS, 2007). Segundo Silva e Barros (2006), as contribuições de Amartya Sen, Anand e Dasgupta têm orientado a construção de indicadores de pobreza multidimensionais, que introduzem a noção de quais as dimensões relevantes, as variáveis adotadas e seus pesos, o método de agregação da pobreza e a forma de agregar a pobreza de todas as pessoas, de foram a analisar o desenvolvimento humano, de foram a construir um indicador escalar sintético de pobreza.

Para Rocha (1998), a pobreza conceitual e operacionalmente relevante depende das condições de vida vigentes e do nível de desenvolvimento em cada sociedade. A pobreza é um fenômeno multidimensional que envolve insuficiência de renda, desigualdade na distribuição da renda produzida socialmente, restrição ao acesso a serviços básicos, à informação, ao trabalho e renda digna, entre outros fatores. 
Para Rocha (2006), as linhas de pobreza expressam o número de indigentes e está relacionada à pobreza absoluta, que considera a necessidade de sobrevivência física e o não atendimento das necessidades nutricionais mínimas essenciais a sobrevivência humana. Já, a pobreza relativa associa-se ao número de pobres de um país e inclui, além das necessidades alimentares, outras necessidades básicas, a exemplo da habitação e do vestuário, as quais devem ser satisfeitas de forma a reduzir as desigualdades existentes no meio social.

O conceito de pobreza absoluta é importante no Brasil porque há muitas pessoas que não têm suas necessidades básicas, nutricionais e biológicas atendidas. As linhas de pobreza adotadas no Brasil, que apresentam uma economia bem monetizada, adotam os valores do salário mínimo ou seus múltiplos, segundo Mocelin (2010, 2011).

O meio rural, segundo Schneider e Fialho (2000), passou por transformações na década de 70, mas nas análises de desenvolvimento dualistas sempre foi considerado mais atrasado e menos desenvolvido que as regiões urbanas. As transformações agrícolas no Brasil na década de 70 aprofundaram a desigualdade e a exclusão social da população rural.

Segundo a Fundação Perseu Abramo (FPA, 2013) do universo de 22,5 milhões de brasileiros que abandonaram a pobreza no último decênio, $74 \%$ deles pertenciam ao meio urbano, enquanto $26 \%$ eram rurais. Por conta disso, o número de pobres urbanos caiu $63,1 \%$ entre 2002 e 2012, enquanto a quantidade de pobres rurais diminuiu 45,8\%. No ano de 2012 , o Brasil registrou 9,8 milhões de pobres urbanos, enquanto em 2002 eram 26,4 milhões de brasileiros vivendo com até 140 reais per capita de rendimento mensal domiciliar. No mesmo período de tempo, a pobreza rural decaiu de 12,8 milhões para 6,9 milhões de pessoas.

Carneiro (2003) verifica a concentração da pobreza nordestina nas áreas rurais. Também destaca a presença maior de pobreza nas áreas urbanas das grandes cidades e das metrópoles nas regiões Sul e Sudeste. O autor destaca que de 1993 a 1998, a pobreza caiu menos no Nordeste, nas áreas rurais, e nas áreas urbanas de pequeno e médio porte. Consequentemente, a pobreza ficou mais concentrada nestas áreas. Esta constatação sugere a necessidade de um foco maior das políticas públicas (infraestrutura, educação, emprego) focadas nos pobres em cidades de pequeno e médio porte e em áreas rurais.

Níger e Silva (2004) mostram que no período de 1995 e 2001 a pobreza nas áreas rurais apresentou queda significativa, com destaque para os estados do Ceará, Rio de Janeiro, Santa Catarina e Goiás. Por outro lado, para o estado de São Paulo, observou-se uma elevação significativa da pobreza nas áreas rurais.

Em seu trabalho sobre fonte de renda rural não agrícola no Brasil. Kageyama (2001) mostrou que as rendas de domicílios agrícolas no Brasil são bastante heterogêneas. A renda domiciliar per capita média de uma família agrícola na região Nordeste ampliada (incluindo Tocantins e Mato Grosso) equivale a um terço daquela observada na região mais rica a citar São Paulo, Mato Grosso do Sul e Minas Gerais. Kageyama (2003) aponta a emergência e crescimento das rendas não agrícolas, em conjunto com as rendas agrícolas para a sobrevivência das famílias ou domicílios rurais, contribuindo para a renda, melhores padrões e consumo e nutrição. Segundo a autora, a diversificação da origem das rendas é uma trajetória que pode permitir a superação do estado de pobreza.

A proporção de pobres é muito maior entre as famílias cujo chefe tem na agricultura sua atividade principal e que possuem domicílio rural. Mesmo os que se concentram nas 
periferias das pequenas e médias cidades, constituindo verdadeiras favelas rurais, estão relativamente em melhor situação que aqueles que moram na zona rural. E dentre os que moram no campo, os que estão ocupados em atividades não agrícolas vivem em melhor situação que os pequenos produtores e trabalhadores rurais temporários (SILVA, 1998).

Ao analisar a pobreza na população do Nordeste, Silva Junior (2006) destaca que o principal determinante da pobreza nordestina é a educação. Outros importantes determinantes da pobreza mais severa e distribuição mais desigual da renda e da pobreza rural são: idade, cor, gênero e atividade principal de ocupação. $\mathrm{O}$ autor destaca a externalidade negativa que a pobreza traz para toda a sociedade, inclusive os não-pobres, ao acentuar as desigualdades existentes, afetando o bem-estar, ao trazer um problema de consciência coletiva.

Para Helfand e Pereira (2012), a pobreza rural no Brasil diminuiu consideravelmente nas últimas décadas, mas continua a ser um problema sério e foco importante das políticas públicas. Grande parte do declínio é atribuída às inovações das políticas de seguridade social e de transferências de renda condicionada, tendo também o Plano Real sido de grande importância.

A pobreza rural está ligada à agricultura familiar e segundo Belik (2000), até 1993 não havia programas e recursos específicos para o financiamento da agricultura familiar no Brasil. Entre as iniciativas que surgiram para combater a pobreza rural na década de 1990 destaca-se o Programa Nacional de Fortalecimento da Agricultura Familiar (PRONAF). Criado em 1994 e tendo deslanchado após 1996, o objetivo do programa era manter as pessoas ocupadas nos estabelecimentos familiares, de forma a gerar renda para remunerar estes postos de trabalho, agregar novas atividades agrícolas e não agrícolas, que induzem o desenvolvimento produtivo e do mercado local (SARTOR et al., 2014).

No início do programa, no governo Fernando Henrique Cardoso, o programa volta-se para a região Sul e tem um pequeno aporte de recursos. No governo Lula, o programa é ampliado substantivamente, em valores e número de contratos, tendo alcançado as demais regiões do país e o Nordeste de forma particular (CAMPOS, 2004). As linhas de crédito estão voltadas para os assentados da reforma agrária, microcrédito para combater a pobreza rural, custeio para os assentados que já recebiam PRONAF, e apoio à agricultura familiar.

Os estudos que analisam os efeitos do PRONAF sobre a economia regional, a produção agrícola e produtividade, como Belik (2000), Mattei (2001, 2005, 2007) e Sartor et al. (2014) destacam o lento crescimento do programa e seus impactos mais significativos no governo Lula e nas diferentes regiões brasileiras. Antunes et al. (2103) analisa os impactos locais no estado do Paraná e verifica o sucesso do programa no incremento da produção agrícola e de animais fomentados pelo programa. Enquanto Mattei et al. (2007) identifica ganhos do PRONAF nas diferentes regiões brasileiras analisadas, inclusive Nordeste, Sudeste e Norte para vários indicadores analisados, Sartor et al. (2014) verificam ganho mais substantivos na região sul. Os resultados de Sartor et al. (2014) sinalizam que o programa pode estar atuando na direção de distribuir renda, sem obter o sucesso esperado no incremento da produção e no desenvolvimento local em outras regiões do país. No entanto, é incontestável que atuou na direção da redução da pobreza rural em todas as regiões ao contribuir para a sustentação da renda da população beneficiada. Batista e Neder (2014) analisam os impactos do PRONAF no Brasil e seus efeitos sobre a pobreza e verificam efeitos indiretos os gastos do Pronaf, pois eles tendem a reduzir indiretamente a pobreza via elevação

Econ. e Desenv., Santa Maria, vol. 27, n.1, p. 183 - 201, jan. - jul. 2015 


\section{Sidnei do Nascimento}

da renda média e da redução da concentração de renda. Os autores também verificam que o efeito do Pronaf sobre a renda per capita média e a desigualdade de renda está condicionado às especificidades socioeconômicas das unidades de observação.

\section{- População rural e taxa de pobreza no Brasil em 2000 e 2010: Evidências empíricas}

Na Tabela 1 é possível observar que entre 2000 e 2010 houve uma redução de $3 \%$ da população rural brasileira, a qual passou de $19 \%$ para 16\%. No ano de 2000 foi observado que a região Nordeste tinha $31 \%$ da população residindo em área rural, no Norte eram em torno de $30 \%$, a região Sul os moradores de áreas rurais representavam 19\% da população total, no Centro-Oeste em torno de $13 \%$ residiam nas zonas rurais e no Sudeste $9,5 \%$.

Já no ano de 2010 a população rural do Nordeste foi de $27 \%$, em comparação ao ano de 2010 houve redução de 4\%. Na região Norte também foi observada a mesma redução de 4 pontos percentuais, e população passou para $26 \%$. No Sul o percentual de morador rural foi de $15 \%$, redução de $4 \%$ se comparado com o ano 2000.

$\mathrm{Na}$ região Centro-Oeste aproximadamente $11 \%$ da população estava residindo em área rural, se comparado com o período anterior houve redução de $2 \%$. A região Sudeste registrou $7 \%$ do total de pessoas residindo em zonas rurais, ou seja, queda de $2,5 \%$ se comparado com o ano de 2000.

A região Nordeste apresentou o maior percentual de população rural nos anos de 2000 e de 2010, já nos mesmos anos as menores taxas foram observadas para a região Sudeste. O Norte, Nordeste e Sul apresentaram as maiores reduções no período analisado se coparado com o Brasil e com as outras regiões.

Tabela 1 - Percentual da população rural no Brasil e Grandes Regiões em 2000 e 2010

\begin{tabular}{l|c|c|c}
\hline Região geográfica & $\begin{array}{c}\mathbf{2 0 0 0} \\
(\mathbf{A})\end{array}$ & $\begin{array}{c}\mathbf{2 0 1 0} \\
(\mathbf{B})\end{array}$ & $(\mathbf{B})-(\mathbf{A})$ \\
\hline Brasil & 19 & 16 & -3 \\
Centro-Oeste & 13 & 11 & -2 \\
Nordeste & 31 & 27 & -4 \\
Norte & 30 & 26 & -4 \\
Sudeste & 9,5 & 7,0 & $-2,5$ \\
Sul & 19 & 15 & -4 \\
\hline
\end{tabular}

Fonte: Elaboração própria com dados do IBGE

Na Tabela 2, no ano de 2000 os estados que apresentavam maior percentual de pessoas residindo em áreas rurais eram Maranhão com $40 \%$ e Piauí com aproximadamente $37 \%$, ambos estados do Nordeste.

$\mathrm{Na}$ região Norte, os estados com maiores percentuais de moradores em áreas rurais são Rondônia com aproximadamente 36\% da população e o Acre onde 34\% residem em áreas rurais. Na região Sul, os estados do Paraná e Rio Grande do Sul contavam com menores percentuais de população rural. Por outro lado, os estados com menores percentuais de

\section{RE\&D Econ. e Desenv., Santa Maria, vol. 27, n.1, p. 183 - 201, jan. - jul. 2015}


população rural estavam na região Sudeste, sendo que Rio de Janeiro com menos de 5\% e o estado de São Paulo em torno de 7\%.

No ano de 2010 os estados do Maranhão (37\%) e Piauí (34\%) apresentaram os maiores percentuais de pessoas morando em área rural se comparado com outros estados. Novamente para o ano de 2010, os estados do Rio de Janeiro, Distrito Federal e São Paulo aparecem com os menores percentuais da população residindo em áreas rurais, a qual se situa abaixo de $5 \%$ da população total.

Tabela 2 - Percentual de população rural e taxa de pobreza nos estados do Brasil em 2000 e 2010

\begin{tabular}{|c|c|c|c|c|c|c|}
\hline Estados & $\begin{array}{c}\text { População } \\
\text { Rural } \\
2000 \\
\text { (A) }\end{array}$ & $\begin{array}{c}\text { Taxa de } \\
\text { Pobreza } \\
2000 \\
\text { (B) }\end{array}$ & $\begin{array}{c}\text { População } \\
\text { Rural } \\
2010 \\
\text { (C) }\end{array}$ & $\begin{array}{c}\text { Taxa de } \\
\text { Pobreza } \\
2010 \\
\text { (D) }\end{array}$ & $(\mathrm{C})-(\mathrm{A})$ & (D) - (B) \\
\hline Acre & 34 & 44 & 27 & 29 & $\overline{-7}$ & -15 \\
\hline Alagoas & 32 & 57 & 26 & 34 & -6 & -23 \\
\hline Amapá & 11 & 38 & 10 & 24 & -1 & -14 \\
\hline Amazonas & 25 & 49 & 21 & 31 & -4 & -18 \\
\hline Bahia & 33 & 50 & 28 & 29 & -5 & -21 \\
\hline Ceará & 28 & 52 & 25 & 30 & -3 & -22 \\
\hline Distrito Federal & 4 & 12 & 3 & 5 & -1 & -7 \\
\hline Espírito Santo & 20 & 23 & 17 & 10 & -3 & -13 \\
\hline Goiás & 12 & 21 & 8 & 8 & -4 & -13 \\
\hline Maranhão & 40 & 63 & 37 & 40 & -3 & -23 \\
\hline Mato Grosso & 21 & 22 & 18 & 11 & -3 & -11 \\
\hline Mato Grosso do Sul & 16 & 23 & 14 & 10 & -2 & -13 \\
\hline Minas Gerais & 18 & 25 & 15 & 11 & -3 & -14 \\
\hline Pará & 33 & 47 & 32 & 32 & -1 & -15 \\
\hline Paraíba & 29 & 50 & 25 & 29 & -4 & -21 \\
\hline Paraná & 19 & 19 & 15 & 6 & -4 & -13 \\
\hline Pernambuco & 23 & 45 & 20 & 27 & -3 & -18 \\
\hline Piauí & 37 & 57 & 34 & 34 & -3 & -23 \\
\hline Rio de Janeiro & 4 & 13 & 3 & 4 & -1 & -9 \\
\hline Rio Grande do Norte & 27 & 45 & 22 & 24 & -5 & -21 \\
\hline Rio Grande do Sul & 18 & 16 & 15 & 6 & -3 & -10 \\
\hline Rondônia & 36 & 30 & 26 & 15 & -10 & -15 \\
\hline Roraima & 24 & 34 & 23 & 27 & -1 & -7 \\
\hline Santa Catarina & 21 & 13 & 16 & 4 & -5 & -9 \\
\hline São Paulo & 7 & 10 & 4 & 5 & -3 & -5 \\
\hline Sergipe & 29 & 49 & 26 & 28 & -3 & -21 \\
\hline Tocantins & 26 & 45 & 21 & 22 & -5 & -23 \\
\hline
\end{tabular}

Fonte: Elaboração própria com dados do IBGE.

É possível destacar ainda que no ano de 2010 houve uma redução no percentual da população morando em áreas rurais, se comparado com o ano de 2000, para todos os estados brasileiros, seguindo uma tendência de aumento da urbanização, com o estado de Rondônia apresentando a maior redução percentual de população rural (10\%).

Ainda na Tabela 2, é possivel destacar que os estados no Norte e Nordeste apresentaram as maiores taxas de pobreza para ambos os anos. No ano de 2000, os estados com maiores taxas de pobreza estavam situados no Nordeste, entre eles estão Alagoas, Ceara, Maranhão e Piauí, todos com mais de $50 \%$ de suas populações vivendo na pobreza. Nos estados em que a taxa de pobreza é de aproximadamente 50\% estão Amazonas, Bahia, 
Paraíba e Sergipe. Os estados da região Sul, Paraná, Santa Catarina e Rio Grande do Sul estão entre os estados com menores taxas de pobreza, a qual se situa abaixo de $20 \%$. O mesmo é observado para os estados de São Paulo, Rio de Janeiro e Distrito Federal.

Para o ano de 2010 houve redução na taxa de pobreza em todos os estados. Os estados das regiões Norte e Nordeste apresentaram as maiores variacões na taxa de pobreza, com reduções maiores que 20 pontos percentuais nos estados de, Alagoas, Bahia, Ceára, Maranhão, Paraíba, Piauí, Rio Grande do Norte, Sergipe e Tocantins. No entando os estados das regiões Norte e Nordeste ainda apresentaram as maiores taxas de pobreza entre os 27 estados da federação.

Os dados da Tabela 2 confirmam os resultados de Helfand e Pereira (2012). Entre os estados brasileiros que apresentam as menores taxas de pobreza, abaixo de $10 \%$, estão Distrito Federal, Goiás, Paraná, Rio Grande do Sul, Rio de Janeiro, Santa Catarina e São Paulo. O resultado aponta na direção proposta por Carneiro (2003). Por outro lado, é possível verificar que a pobreza cai menos na região Norte e entre os fatores a concentração da posse da terra ${ }^{1}$ e o menor atendimento do PRONAF nesta região também pode estar entre os fatores que contribuíram para tais resultados, conforme salientado por Sartor et al. (2014), Batista e Neder (2014) e Belik (2010).

Batista e Neder (2015) estudaram os efeitos do PRONAF sobre a pobreza rural no Brasil no período de 2001-2009. As estimativas sugerem que uma elevação em $10 \%$ do crédito do PRONAF tende a elevar a renda per capita domiciliar rural em $0,24 \%$ e a reduzir a desigualdade de renda em $0,36 \%$. Os valores apresentam, portanto, a incidência indireta da variável PRONAF na redução da pobreza em duas vias: pelo aumento da renda domiciliar per capita e pela melhor distribuição de renda mensurada pelo Índice de Gini.

Já, Sartor et al. (2014) destacam que o número de contratos do PRONAF por regiões no ano de 2000 foi: Sul (550 mil), Sudeste (150 mil), Centro-Oeste (40 mil), Nordeste (300 mil) e Norte (10 mil). Para o ano de 2009, o número de contratos por região foi: Sul (600 mil), Sudeste (250 mil), Centro-Oeste (50 mil), Nordeste (450 mil) e o Norte (40 mil).

\section{PROCEDIMENTOS METODOLÓGICOS}

Nesta seção é abordada a metodologia utilizada na pesquisa, a descrição das variáveis utilizadas e os correspondentes bancos de dados.

\section{- Análise Exploratória de Dados Espaciais (AEDE)}

A análise exploratória de dados espaciais é a técnica para descrever e visualizar distribuições espaciais, identificar localidades espaciais atípicas, descobrir padrões de associação espacial e sugerir diferentes regimes espaciais. O primeiro passo no estudo de AEDE é testar a hipótese de que os dados espaciais sejam distribuídos aleatoriamente.

\footnotetext{
${ }^{1}$ Aquino e Lacerda (2014) mostram que o Rio Grande do Norte tinha 42 mil estabelecimentos rurais no ano de 2006, dentre os produtores estudados 54,5\% tem menos de cinco hectares de terra para trabalhar e viver, $19,5 \%$ dos produtores tinham estabelecimentos entre 10 e 50 ha, 12,5\% tinham áreas inferiores a 10 ha, em torno de 4,3\% tinham entre 50 e 100 ha, 1,2\% dos estabelecimentos dispunha de extensões de terra acima de 100 ha e aproximadamente $8 \%$ estavam enquadrados como produtor sem terra.
} 
Intuitivamente, a aleatoriedade espacial indica que os valores de um atributo numa região não dependem dos valores deste atributo nas regiões vizinhas (ALMEIDA, 2012).

No entanto essa análise é mais apropriada na investigação de variáveis espacialmente densas ou intensivas, ou seja, variáveis que são divididas por algum indicador de intensidade (per capita, etc.). Nesse sentido, Perobelli et al. (2007) reforçam que a partir da AEDE é possível extrair medidas de autocorrelação espacial global e local, investigando a influência dos efeitos espaciais por intermédio de métodos quantitativos.

\section{- Matrizes de Pesos Espaciais}

O conceito de matrizes de pesos espaciais é baseado na proximidade, que, por sua vez, pode ser definida de acordo com a vizinhança, a distância tanto geográfica quanto socioeconômica, bem como um a combinação destas. A discussão a respeito da tipologia das matrizes é questão importante e delicada na literatura, das formas de matrizes de pesos espaciais utilizadas as mais comuns são as convenção rainha e torre (ALMEIDA, 2012). Ambas as matrizes são apresentada na Figura 1.

Figura 1 - Matrizes de contiguidade

\section{Rainha}

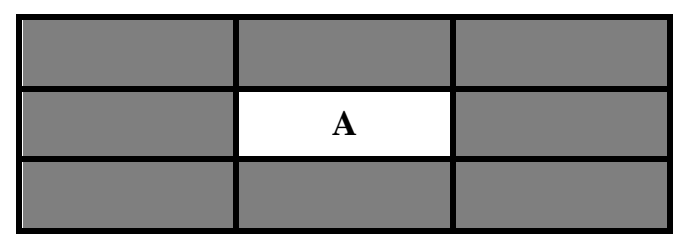

Fonte: Elaborado pelos autores com base em Almeida (2012)
Torre

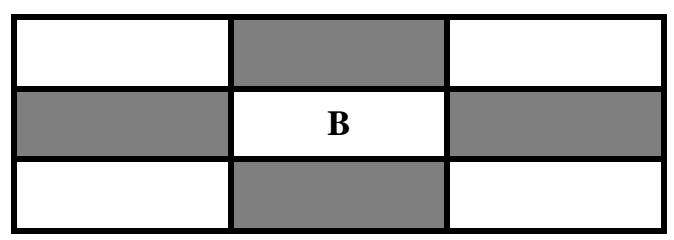

A convenção de contiguidade é dita ser rainha, caso além das fronteiras com extensão diferente de zero, puderem ser considerados os vértices (nós), na visualização de um mapa, como contíguos. Caso apenas as fronteiras físicas com extensão diferente de zero entre as regiões sejam levadas em conta, a convenção de contiguidade é considerada como torre. Essas duas convenções são as mais utilizadas na literatura.

\section{- A Estatística I de Moran}

A estatística I de Moran é utilizada para verificar a existência de autocorrelação espacial de uma variável em uma determinada região. Foi proposta por Patrick A. P. Moran no ano de 1948. Algebricamente a estatística I de Moran é representada pela equação 1:

$$
\mathrm{I}=\frac{\mathrm{n}}{\mathrm{S}_{0}} \frac{\sum_{\mathrm{i}} \sum_{\mathrm{j}} \mathrm{w}_{\mathrm{ij}} \mathrm{z}_{\mathrm{i}} \mathrm{z}_{\mathrm{j}}}{\sum_{\mathrm{i}=1}^{\mathrm{n}} \mathrm{z}^{2}}
$$

Ou matricialmente, a equação 1 é representada pela equação 2:

$$
\mathrm{I}=\frac{\mathrm{n}}{\mathrm{S}_{0}} \frac{\mathrm{z}^{\prime} \mathrm{Wz}}{\mathrm{z}^{\prime} \mathrm{z}}
$$

Econ. e Desenv., Santa Maria, vol. 27, n.1, p. 183 - 201, jan. -jul. 2015 
Em que $n$ é o número de regiões, $z$ denota os valores da variável de interesse padronizada, $W z$ representa os valores médios da variável de interesse padronizada nos vizinhos, definidos segundo uma matriz de ponderação espacial $W$. Um elemento dessa matriz, referente à região $i$ e a região $j$, é registrado como $w_{i j}$, significando que todos os elementos da matriz de pesos espaciais $w$ devem ser somados.

Os valores de $i$ maiores (ou menores) do que o valor esperado de $\mathrm{E}(\mathrm{I})=[-1 /(\mathrm{n}-1)]$ significa que há autocorrelação positiva (ou negativa). De acordo com Almeida (2012), a autocorrelação espacial positiva revela que existe uma similaridade entre os valores do atributo estudado e da localização espacial do atributo. A autocorrelação espacial negativa revela, por sua vez, que existe uma dissimilaridade entre os valores do atributo considerado e a localização espacial.

Segundo Almeida (2012), o I de Moran fornece três tipos de informações: 1) o nível de significância fornece a informação sobre os dados estarem distribuídos aleatoriamente ou não; 2) o sinal positivo da estatística I de Moran, desde que significativos, indica que os dados estão concentrados em regiões. O sinal negativo, por sua vez, indica a dispersão dos dados; 3 ) a magnitude da estatística fornece a força da autocorrelação espacial, quanto mais próximo de um mais forte é autocorrelação e quanto mais próximo de -1 mais disperso estão os dados.

\section{- I de Moran Global Bivariado}

A autocorrelação espacial global bivariada permite verificar se uma variável observada em determinada região tem alguma associação com outra variável em regiões vizinhas. O coeficiente I de Moran bivariado é dado pela equação abaixo:

$$
\mathrm{I}^{\mathrm{Z}_{1} \mathrm{Z}_{2}}=\frac{\mathrm{n}}{\mathrm{S}_{0}} \frac{\mathrm{Z}_{1}^{\prime} \mathrm{WZ}}{\mathrm{Z}_{1}^{\prime} \mathrm{Z}_{1}}
$$

Onde $\mathrm{WZ}_{2}$ representa a defasagem da variável $\mathrm{Z}_{2}$. A presença de autocorrelação espacial positiva indica uma associação dos valores das variáveis que está sendo estudada (nesse caso o percentual da população rural e a taxa de pobreza) e de suas localizações. Sendo assim, a autocorrelação positiva mostra que municípios com uma alta (baixa) população rural são rodeados por municípios com uma alta (baixo) taxa de pobreza.

O I de Moran esperado é dado por E (I) $=[-1 /(n-1)]$, fornece o valor que seria obtido se não houvesse padrão espacial nos dados. Sendo que os valores de I de Moran acima da esperança de Moran indicam autocorrelação espacial positiva e os valores abaixo indicam autocorrelação negativa.

\section{- O Diagrama de Dispersão de Moran}

Segundo Almeida (2012), o diagrama de dispersão de Moran é uma alternativa para visualizar a autocorrelação espacial, o qual mostra a defasagem espacial da variável de interesse no eixo vertical e o valor da variável de interesse no eixo horizontal. Além da medida global de associação linear espacial, o diagrama dispersão mostra associação espacial entre as regiões e seus vizinhos dividido em quatro quadrantes: alto-alto (AA), baixo-baixo (BB), alto-baixo (AB) e baixo-alto (BA), como mostra a Figura 2:

\footnotetext{
RE\&D Econ. e Desenv., Santa Maria, vol. 27, n.1, p. 183 - 201, jan. - jul. 2015
} 
Figura 2 - Diagrama de dispersão de Moran
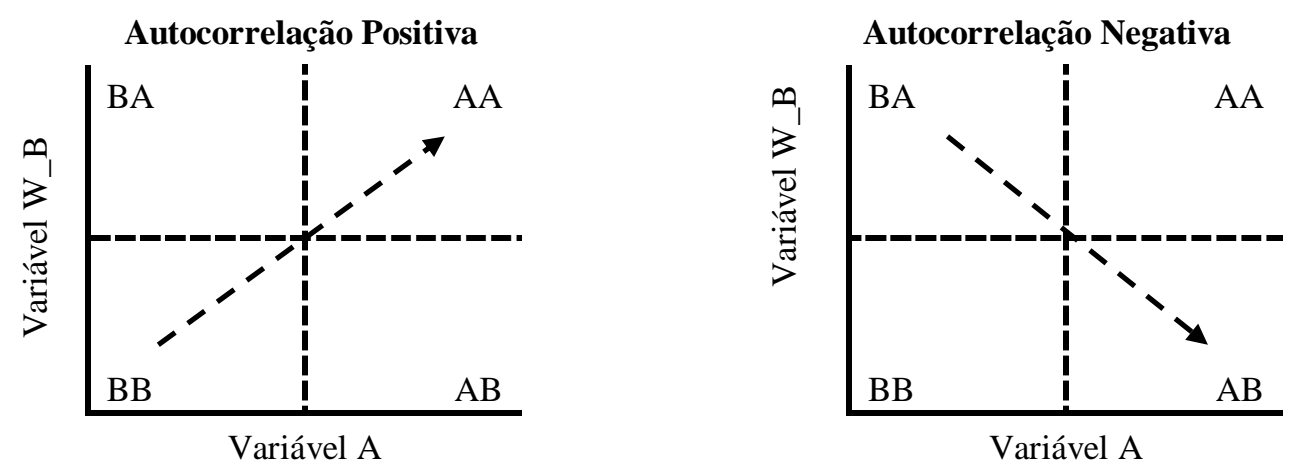

Fonte: Elaborado pelos autores com base em Almeida (2012)

Um agrupamento alto-alto (AA) significa que as unidades espaciais pertencentes a esse agrupamento exibem valores altos da variável de interesse (A) rodeados por unidades espaciais que apresentam valores também altos da variável defasada (B), representado pelo primeiro quadrante do diagrama. Um agrupamento baixo-alto (BA) concerne a um cluster no qual uma unidade espacial qualquer com um baixo valor da variável de interesse (A) são circundados por unidades espaciais com alto valor da variável (B). Isso é representado no segundo quadrante.

Um agrupamento baixo-baixo (BB) refere-se a um agrupamento cujas unidades espaciais mostram valores baixos da variável (A) circundados por unidades espaciais que ostentam valores também baixos da defasagem da variável (B), representado pelo terceiro quadrante. Um agrupamento alto-baixo $(\mathrm{AB})$ diz respeito a um cluster no qual uma unidade espacial qualquer com um alto valor da variável de interesse (A) são vizinhos de unidades espaciais com um baixo valor da variável (B). Isso é representado pelo quarto quadrante.

\section{- Análise Bivariada Local}

Segundo Almeida (2012), assim como se pôde obter um coeficiente de autocorrelação espacial global num contexto bivariado, também é possível conseguir uma medida de autocorrelação espacial local multivariada. Readaptando o I de Moran local obtemos a equação 4 :

$$
\mathrm{I}_{\mathrm{i}}^{\mathrm{Z}_{1} \mathrm{Z}_{2}}=\mathrm{Z}_{1 \mathrm{i}} \mathrm{WZ}_{2 \mathrm{i}}
$$

Em que $w_{2} z_{2 i}$ é a defasagem espacial da variável padronizada $z_{2 i}$. Semelhantemente à fórmula do calculo do I de Moran na equação 3, somente os vizinhos da observação $i$, definidos conforme uma matriz de pesos espaciais, são incluídos no cálculo.

De acordo com Anselin et al. (2003, apud Almeida, 2012), essa estatística dá uma indicação do grau de associação linear (positiva ou negativa) entre o valor para uma variável em uma dada locação $i$ e a média de uma outra variável nas locações vizinhas. É possível mapear os valores da probabilidade da medida, estatisticamente significativos, gerando o chamado mapa de significância bivariado do Moran local. 


\section{- Descrição das variáveis e bases de dados}

A base de dados esta disponível no site do Instituto Brasileiro de Geografia e Estatística (IBGE), censos de 2000 e 2010 e do Instituto de Pesquisa Economia Aplicada (IPEA). Os dados utilizados são: percentual da população rural e taxa de pobreza dos municípios brasileiros de 2000 e 2010.

Para mensurar o percentual da população rural utilizou-se a divisão simples da população rural pela população total multiplicado por 100. Para a variável taxa de pobreza, utilizou-se proporção dos indivíduos com renda domiciliar per capita igual ou inferior a R \$ 140,00 mensais, em reais de agosto de 2010 para os municípios brasileiros. O universo de indivíduos é limitado àqueles que vivem em domicílios particulares permanentes.

\section{ANÁLISE DOS RESULTADOS}

Nesta secção são apresentados os resultados encontrados da análise exploratória de dados espaciais das seguintes variáveis: percentual da população rural e taxa de pobreza nos municípios brasileiros dos anos de 2000 e de 2010.

\section{- I de Moran global bivariado}

Na Tabela 3 são apresentados os resultados para autocorrelação global bivariada, em que foi utilizada uma matriz de pesos espaciais do tipo Rainha. Os valores do coeficiente I de Moran para a variável percentual de população rural e taxa de pobreza no ano de 2000 e de 2010 ficou acima do valor esperado de - 0,0002, os valores de 0,37 no ano de 2000 e de 0,38 no ano de 2010 e significativo a $1 \%$ indicam que nos dois anos analisados existe autocorrelação positiva entre as variáveis.

Tabela 3 - Autocorrelação Global Bivariado do percentual de população rural e taxa de pobreza dos municípios do Brasil no ano de 2000 e de 2010

\begin{tabular}{c|c|c}
\hline Variável & I de Moran & Probabilidade \\
\hline W_TX_POB x POP_RUR 2000 & 0,3724 & 0,001 \\
W_TX_POB x POP_RUR 2010 & 0,3842 & 0,001 \\
\hline
\end{tabular}

Fonte: Elaboração própria com dados do IBGE e utilizando o software Geoda

Verifica-se que ao longo do período analisado, ano de 2000 e de 2010, ocorre um aumento pouco expressivo na autocorrelação espacial, ou seja, tem-se quase o mesmo padrão de concentração espacial entre o percentual da população rural e taxa de pobreza dos municípios.

Os resultados observados na Tabela 3 revelam a existência de regiões com quantidade elevada de pessoas residindo em área rural e com taxa de pobreza acima da média, reforçando as evidências apresentadas no relatório do Banco Mundial (2008) que mostram que a pobreza é um fenômeno generalizado e continua a atingir com mais intensidade as pessoas que vivem em áreas rurais. 
Outra forma de visualizar a autocorrelação espacial é através do diagrama de dispersão de Moran, o qual é dividido em quatro quadrantes demonstrando o padrão de agrupamento dos municípios analisados (DINIZ, 2012).

\section{- Diagrama de dispersão de Moran bivariado}

O resultado do diagrama de Moran bivariado (Figura 3) relaciona o percentual de população rural (plotado no eixo $x$ ) e a taxa de pobreza (colocada no eixo $y$ ) dos municípios brasileiros no ano de 2000 e de 2010. O diagrama é dividido em quatro categorias, alto-alto (AA), baixo-baixo (BB), baixo-alto (BA) e alto-baixo (AB). A relação positiva entre as variáveis é reforçada com a inclinação ascendente da reta de regressão, ou seja, a tendência de concentração dos municípios no primeiro e no terceiro quadrante.

O diagrama de dispersão referente ao ano de 2000 mostra que $31 \%$ dos municípios analisados encontram-se no primeiro quadrante, ou seja, são regime espacial alto-alto (AA), em torno de $35 \%$ estão concentrados no terceiro quadrante, sendo assim são aglomerações espaciais baixo-baixo (BB). As regiões atípicas são representadas por $34 \%$ das unidades municipais, sendo que $15 \%$ estão localizadas no segundo quadrante e outras $19 \%$ no quarto quadrante.

No ano de 2010 o diagrama de Moran revela que 29\% dos municípios analisados estão no primeiro quadrante, em torno de $36 \%$ estão no terceiro quadrante. No que tange as regiões atípicas, foi observada que $14 \%$ das unidades municipais se encontram no segundo quadrante e $21 \%$ estão situadas no quarto quadrante.

O padrão de distribuição observado nos diagramas de dispersão corrobora os resultados encontrados com o I de Moran, ou seja, quantidade significativa de municípios brasileiros apresenta taxa de pobreza abaixo da média e são cercados por unidades espaciais com baixo percentual de pessoas residindo em área rural. Por outro lado, os municípios concentrados no primeiro quadrante contemplam elevada taxa de pobreza e a quantidade de pessoas residindo em área rural esta acima da média.

As regiões atípicas representam as unidades espaciais onde as variáveis apresentam dissimilaridades, sendo assim, os municípios situados no segundo quadrante exibem taxa de pobreza abaixo da média e estão cercados por unidades espaciais com baixo percentual de população rural e no quarto quadrante estão às localidades espaciais com elevada taxa de pobreza, no entanto, são circunvizinhados por unidades com baixo percentual de população rural, nesse caso, a pobreza pode estar sendo ocasionada por outros fatores, segundo Barros, Henrique e Mendonça (2001) a pobreza esta associada com a renda concentrada, Rocha (2006) destaca o baixo nível educacional.

Figura 3 - Diagrama de Dispersão de Moran Bivariado da Taxa de População Rural e Taxa de Pobreza para os Anos de 2000 e 2010 


\section{Sidnei do Nascimento}
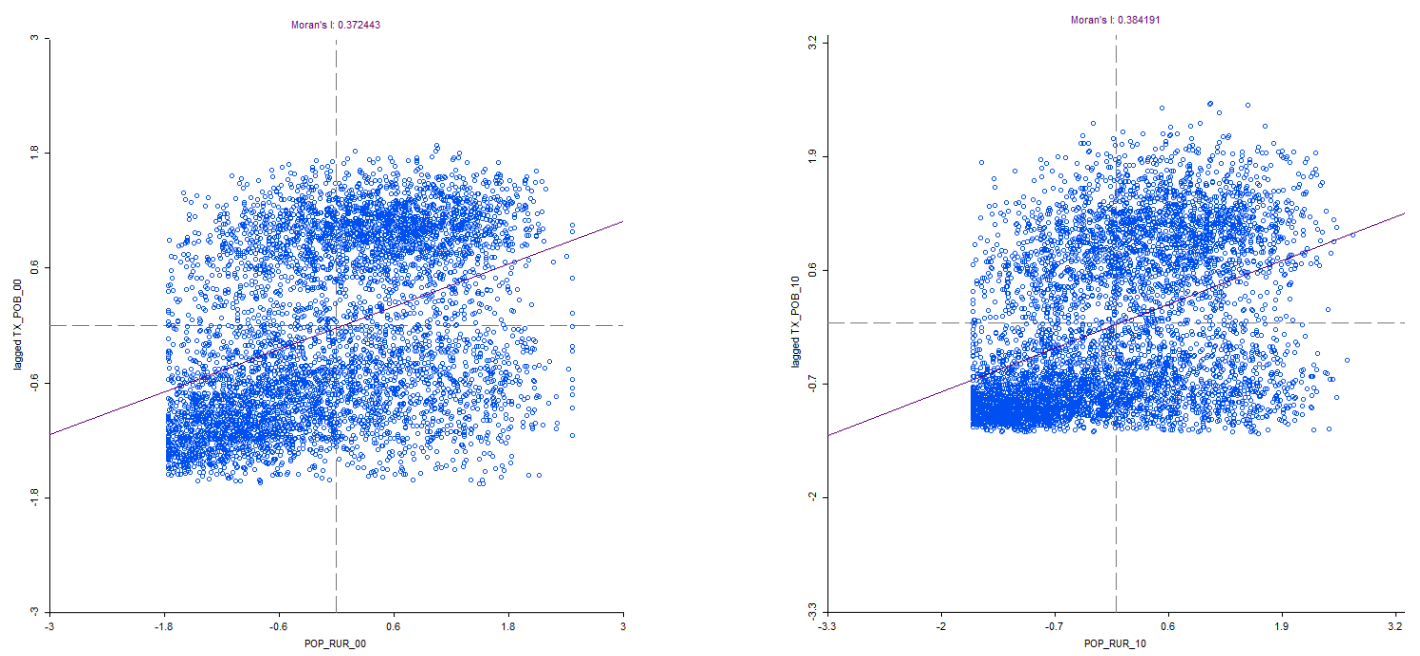

Fonte: Elaboração própria com dados do IBGE e utilizando o Software Geoda

Além do gráfico de dispersão, que exibe todas as diversas regiões compreendidas no estudo, é possível mapear os resultados estatisticamente significantes, para isto, pode ser utilizado o ferramental LISA. Trata-se de mapear as regiões em cada regime discutido pelo gráfico de dispersão de Moran, considerando, entretanto, a hipótese de que, a um grau de confiança de 5\%, nem todas as unidades regionais pertencem de maneira estatisticamente significante a cada um dos regimes considerados (PIMENTEL; HADDAD, 2004).

\section{- Mapa de significância e de cluster bivariado}

O mapa de Significância (LISA) exibido na Figura 4 possibilita observar onde foram formados os clusters estatisticamente significativos da relação entre população rural e taxa de pobreza no ano de 2000. Combinando as informações do diagrama de dispersão de Moran (Figura 3) com o mapa de significância local obtém-se o mapa de cluster, o qual apresenta quatro categorias estatisticamente significativas.

No mapa Lisa as regiões destacadas em verde escuro representam os regimes estatisticamente significativos a $0,1 \%$ e os municípios destacados em verde mais claro as associações significativas a 5\%. Na Figura 4, do total analisado, 1.248 municípios brasileiros apresentaram significância pelo menos a 5\%, em torno de 1.300 unidades espaciais são significativas a $1 \%$ e outras 1.063 localidades municipais foi significância a $0,1 \%$.

No mapa de cluster para o ano de 2000 a associação espacial alto-alto (AA) aparece localizada nas regiões Norte e Nordeste, ou seja, são regiões que exibem alto percentual de população residindo em área rural e cercada por municípios vizinhos que exibem elevada taxa de pobreza. Foi observado nessas regiões considerável formação de agrupamento baixo-alto (BA), deixando evidente a presença de localidades municipais com baixo percentual de população rural, entretanto com municípios vizinhos apresentando taxa de pobreza acima da média.

Por outro lado, o regime espacial baixo-baixo (BB) está concentrado em municípios das regiões Sudeste, Centro-Oeste e Sul do país, demonstrando aglomerações de municípios que apresentaram baixo percentual de pessoas morando na área rural e circunvizinhada por municípios que ostentam baixa taxa de pobreza. Aparecem em destaque nas regiões Sul, 
Sudeste e Centro-Oeste a formação de aglomeração espacial alto-baixo (AB), ou seja, são unidades que apresentam elevado percentual de população rural, porém, está cercado por municípios com baixa taxa de pobreza.

Figura 4 - Mapa Lisa e de Cluster Bivariado da População Rural e Taxa de Pobreza de 2000

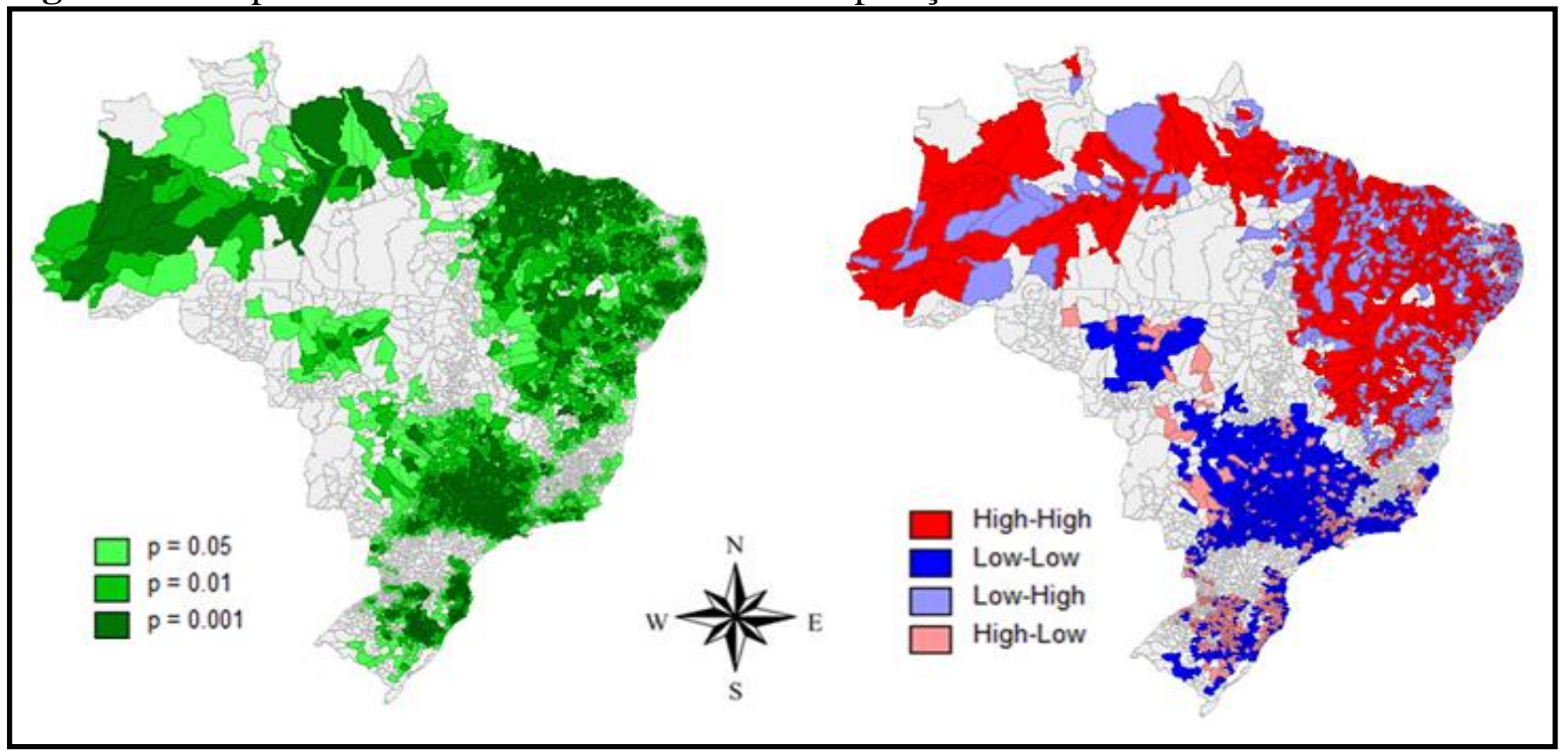

Fonte: Elaboração própria com dados do IBGE e utilizando o Software Geoda

Os clusters estatisticamente significativos formados a partir da relação entre percentual de população rural e taxa de pobreza dos municípios do Brasil no ano de 2010 é apresentado na Figura 5.

No mapa de significância (Figura 5) aproximadamente 1.263 municípios apresentaram significância a 5\%, as unidades espaciais estatisticamente significativas a $1 \%$ somaram em torno de 1.261 e outras 1.092 municípios apresentaram significância a $0,1 \%$.

No mapa de cluster do ano de 2010 a associação espacial alto-alto (AA) continuou concentrada nas regiões Norte e Nordeste. Tal resultado evidência que naquelas regiões a taxa de pobreza se manteve acima da média nacional, e o percentual de população rural dos municípios é elevada. Está em destaque nas mesmas regiões a formação de cluster baixo-alto (BA), ou seja, municípios com baixa percentual de pessoas residindo em área rural, porém cercados por vizinhos com elevada taxa de pobreza.

$\mathrm{O}$ regime espacial baixo-baixo $(\mathrm{BB})$ aparece concentrado em municípios da região Sul, Sudeste e Centro-Oeste, o que demonstra aglomerações de municípios que apresentaram baixo percentual de pessoas morando na área rural e circunvizinhados por unidades municipais com baixa taxa de pobreza. O agrupamento alto-baixo (AB) denotado em vermelho mais claro no mapa revela a presença de municípios com baixo percentual de população rural, mas os vizinhos apresentam taxa de pobreza acima da média, estão em destaque no mapa unidades pertencentes às regiões Sul, Sudeste e Centro-Oeste (Figura 5).

Se por um lado, o PRONAF pode estar atenuando a pobreza rural de forma acentuada na região Sul e menor grau na região Nordeste, o menor acesso dos municípios da região Norte pode estar contribuindo para a pequena mudança verificada na localização da pobreza rural e na sua relação com pequenos municípios, conforme proposto por Sartor et al. (2014), Mattei (2001, 2007), Antunes et al. (2013) e Kageyama (2006).

Econ. e Desenv., Santa Maria, vol. 27, n.1, p. 183 - 201, jan. - jul. 2015 
Figura 5 - Mapa Lisa e de Cluster bivariado da população rural e taxa de pobreza de 2010

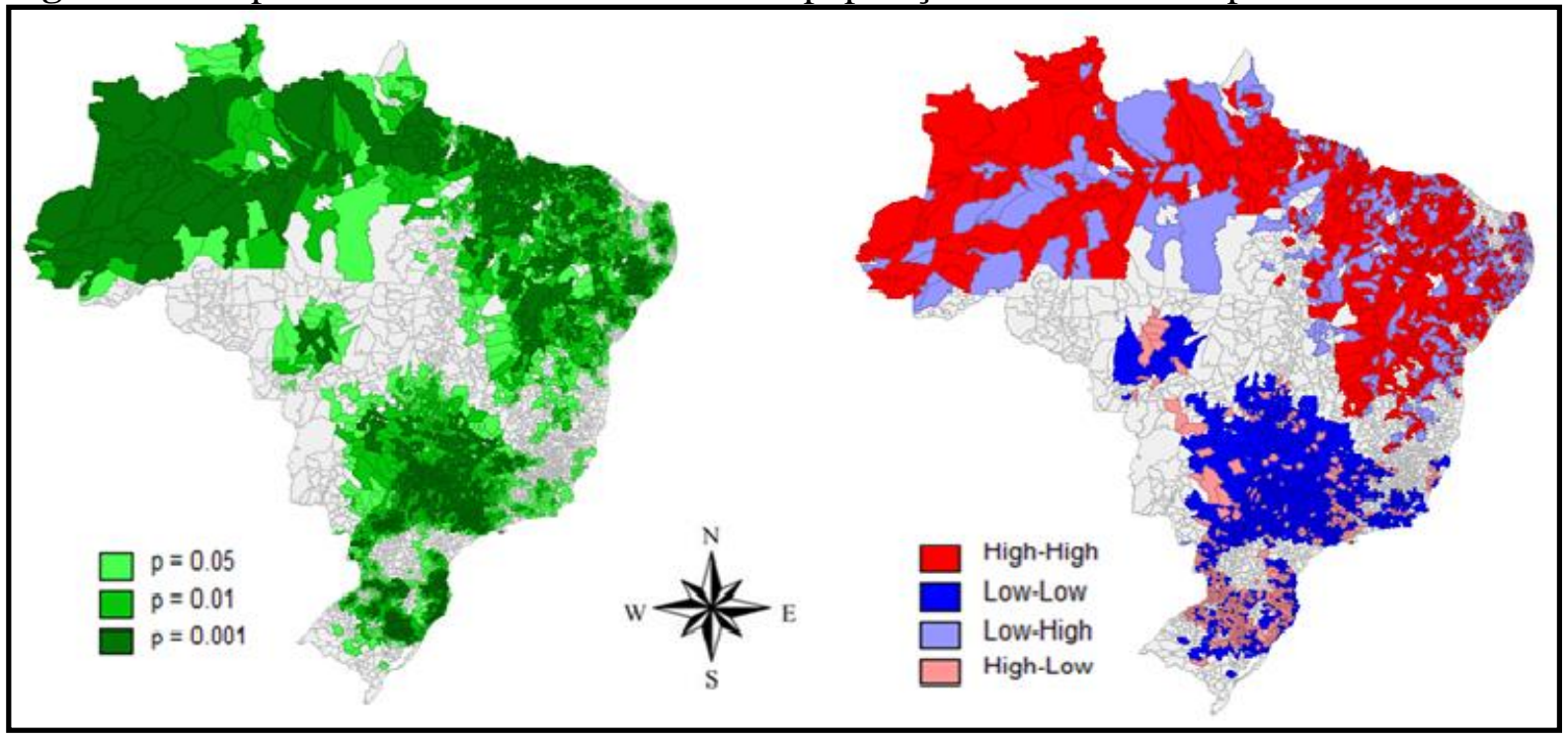

Fonte: Elaboração própria com dados do IBGE e utilizando o Software Geoda

Pode-se destacar nos dois períodos analisados, que as localidades municipais com altas taxas de população rural são vizinhas de unidades com altos percentuais de pobreza, e as regiões com baixas taxas de população rural, apresentam baixo percentuais de pobreza. A aglomeração espacial alto-alto (AA) aparece concentrada nas regiões Norte e Nordeste, enquanto que o regime espacial baixo-baixo (BB) está localizado nas regiões Sul, Sudeste e Centro-Oeste.

Os estudos referentes ao PRONAF indicaram o sucesso do programa na região Sul ${ }^{2}$, em termos de incremento da produção e da renda agrícola de assentados e agricultores familiares, conforme Sartor et al. (2014), Mattei (2001, 2005, 2007) e Antunes et al. (2013). Os resultados obtidos confirmam os dados de Helfand e Pereira (2012) para a pobreza rural no Nordeste no período 2000 e 2010 pela análise comparativa dos mapas nas Figuras 4 e 5.

\section{CONSIDERAÇÕES FINAIS}

Com os resultados desse estudo pode ser verificado que o padrão locacional entre o percentual da população rural e taxa de pobreza para os municípios brasileiros, verificando a autocorrelação espacial e a presença de clusters espaciais entre os municípios. Através da análise exploratória de dados espaciais identificou-se que, em geral, os municípios com alto percentual de população pobre e alta taxa de pobreza são vizinhos de outros municípios com a mesma característica, assim como os municípios com baixa população rural e baixa taxa de pobreza são cercados por municípios nas mesmas condições.

\footnotetext{
${ }^{2}$ Antunes et al. (2013) estudaram os impactos do PRONAF na economia de 60 municípios do estado do Paraná que contrataram crédito entre os anos de 2000 e de 2005. Os resultados encontrados pelos autores revelaram que uma expansão dos recursos do PRONAF provoca efeitos positivos sobre os indicadores de produção (PIB agropecuário, área plantada, quantidade produzida), receita tributária e emprego formal agropecuário.
}

RE\&D Econ. e Desenv., Santa Maria, vol. 27, n.1, p. 183 - 201, jan. - jul. 2015 
Pode-se verificar através da análise exploratória de dados espaciais, que entre 2000 e 2010 houve um aumento pouco expressivo da autocorrelação positiva entre o percentual da população rural e taxa de pobreza para os municípios brasileiros.

Com os resultados da Análise Exploratória de Dados Espaciais (AEDE), pode-se apontar a concentração de municípios com os maiores valores do percentual da população rural e taxa de pobreza nas regiões Norte e Nordeste, e a concentração de municípios com os menores valores das variáveis nas regiões Sul e Sudeste. Visto que não houve um aumento muito significante do número de municípios do tipo alto-alto e baixo-baixo no período, e também não apresentou uma redução muito grande nas regiões atípicas (alto-baixo e baixoalto). Os estudos apontam para novas pesquisas na direção de políticas para mitigar a pobreza rural, a exemplo dos efeitos municipais do PRONAF.

Portanto, concluem-se que, mesmo havendo uma redução significativa da taxa de pobreza no período de 2000 a 2010, como mostram os dados do IBGE e redução da população rural como revelam os dados do IPEA no mesmo período, as cidades que concentram uma grande população rural continuam com uma alta taxa de pobreza o que confirma a ideia de que a população rural tem dificuldade em usufruir de políticas de combate à pobreza devido à dificuldade de ter acesso a essas informações e pelo distanciamento físico.

\section{REFERÊNCIAS}

ALMEIDA, E. Econometria Espacial. Campinas, SP: Editora alínea. 2012.

ANTUNES, R.L.; CAMARA, M.R.G.; NASCIMENTO, S.P.; SEREIA, V.J.; ANHESINI, J.A.R. Programa nacional de crédito da agricultura familiar e impactos nas economias locais no estado do Paraná. Economia \& Região, Londrina-PR, v.1, n.1, p.69-90, jan./jul. 2013.

AQUINO J.R.; LACERDA, M.A.D. Magnitude e Condições de Reprodução Econômica dos Agricultores Familiares Pobres no Semiárido Brasileiro: evidências a partir do Rio Grande do Norte. RESR, Piracicaba-SP, vol. 52, Supl. 1, p. 167-188, 2014.

BARROS, R.P.; HENRIQUES, R.; MENDONÇA, R. A estabilidade inaceitável:

Desigualdade e pobreza no Brasil. Brasília: IPEA, Texto para discussão $n^{\circ} 800,2001$.

BELIK, W. PRONAF: avaliação da operacionalização do programa. In: CAMPANHOLA, Clayton; GRAZIANO DA SILVA, J. (Org.). O Novo Rural Brasileiro Políticas Públicas. Jaguariúna: EMBRAPA Meio Ambiente, 2000, v. 4, p. 93-115.

CAMPOS, R.F. PRONAF: índices de financiamento e características da distribuição do crédito rural no Brasil de 1996 a 2001. 2004. Dissertação (mestrado), UNICAMPFaculdade de Engenharia Agrícola. Campinas: SP, 2004.

CARNEIRO, F.G. Perfil da pobreza e aspectos funcionais dos mercados de trabalho no Brasil. In CEPAL. Pobreza e mercados no Brasil. Brasília, 2003.

CEPEA - CENTRO DE ECONOMIA E PESQUISA ECONÔMICA APLICADA. Disponível em: www.cepea.esalq.usp.br. Acesso em: 25 de agosto de 2015.

DINIZ, S.S. Analise espacial da produtividade da Laranja dos municípios do estado de

São Paulo: 2002 a 2010. Dissertação de Mestrado. Universidade Estadual de Londrina, 2012, $118 \mathrm{p}$. 
FPA - FUNDAÇÃO PERSEU ABRAMO. Os intocáveis I: A queda da pobreza no Brasil no último decênio. Comunica 6, out. 2013. Disponível em:

http://novo.fpabramo.org.br/sites/default/files/fpa_comunica_06.pdf. Acesso em: 26 Agosto de 2015.

HELFAND, S.M.; PEREIRA, V.F. Determinantes da pobreza rural e implicações para a política pública no Brasil. In: MIRANDA, C.; TIBURCIO, B. A nova cara da pobreza rural: desafios para as políticas públicas. Brasília: IICA, p. 154:160, 2012.

IBGE - INSTITUTO BRASILEIRO DE GEOGRAFIA E ESTATÍSTICA. Disponível em http: //www.ibge.com.br, Instituto Brasileiro de Geografia e Estatística (IBGE). Acessado em 25 de novembro de 2013.

IPEA - INSTITUTO DE PESQUISAS ECONÔMICAS. Disponível em http://www.ipea.gov.br, Instituto de Pesquisa Econômica Aplicada (IPEA). Acesso em 26 de novembro de 2013.

KAGEYAMA, A. As múltiplas fontes de renda das famílias agrícolas brasileiras. Agricultura em São Paulo, São Paulo-SP, v. 48. n. 2, p. 57-69, 2001.

Diversificação das rendas nos domicílios agrícolas no Brasil, 1992 e 2001.

Economia e Sociedade, Campinas-SP, v. 12, n. 1 (20), p. 65-86, jan./jun. 2003.

MATTEI, L.; WAQUIL, P.; SCHNEIDER, S.; CONTERATO, M.; RADOMSKY, G.;

NIEDERLE, S.Uma análise dos impactos do PRONAF sobre as economias locais nas regiões Nordeste, Sudeste e Norte do Brasil. Anais do XLV Congresso da SOBER, 2007.

MATTEI, L. Impactos do PRONAF: Análise de indicadores. Ministério do Desenvolvimento Agrário, NED. Brasília, 2005.

MATTEI, L. Programa Nacional de Fortalecimento da Agricultura Familiar (PRONAF): Concepção, abrangência e limites observados. Anais do IV Encontro da Sociedade Brasileira de Sistemas de Produção, Belém, p. 1-15, 2001. Disponível em: < http://gipaf.cnptia.embrapa.br/publicacoes/artigos-e-trabalhos/trabalhosbsp3.pdf>. Acessado em: 13 de fevereiro de 2010.

MOCELIN, C.E. O Programa Bolsa Família enquanto principal estratégia de enfrentamento à pobreza rural no contexto brasileiro atual. In: XV Seminário Interinstitucional de Ensino, Pesquisa e Extensão da Unicruz. Universidade de Cruz Alta, novembro de 2010. Disponível em: 〈http://www.unicruz.edu.br〉. Acesso em 05 de Dezembro de 2013.

Pobreza rural e o Programa Bolsa Família: o caso dos beneficiários da comunidade de São João do Barro Preto - Júlio de Castilhos. RS. Dissertação de Mestrado. Universidade Federal de Santa Maria. - Programa de Pós-graduação em Extensão Rural, 2011. Disponível em http://cascavel.ufsm.br/tede/tde_busca/arquivo.php?codArquivo=4349. Capturado em 13 de outubro de 2014.

NEDER, H.D.; SILVA, J.L.M. Pobreza e distribuição de renda em áreas rurais. Uma abordagem de inferência. RER, Rio de Janeiro, vol. 42, nº 03, p. 469-486, jul/set 2004.

PEROBELLI, F.S.; ALMEIDA, E.S.; ALVIM, M.I.S.A.; FERREIRA, P.G.C. Análise espacial da produtividade do setor agrícola brasileiro: 1991-2003. Nova economia. Belo Horizonte-MG, vol.17, no. 1, p. 65-91, Jan./Apr. 2007. 
PIMENTEL, E.; HADDAD, E.A. Análise da distribuição espacial da renda no estado de Minas Gerais: Uma abordagem setorial. Núcleo de Economia Regional e Urbana do Estado de São Paulo. Fevereiro de 2004.

ROCHA, S. Pobreza no Brasil: afinal do que se trata? Rio de Janeiro, Ed. FGV, 2006.

Renda e Pobreza: Medidas per capita versus adulto-equivalente. IPEA/DIPES. Abril, 1998.

SANTOS, L.M. Pobreza como privação de liberdade: Um estudo de caso na favela do Vidigal no Rio de Janeiro. (Dissertação de Mestrado) Rio de Janeiro, Universidade Federal Fluminense. Pós-Graduação em Economia, 2007.

http://www.cpgeconomia.uff.br//novosite/arquivos/tese/2007-larissa_martins.pdf.

SARTOR, M.J.; CAMARA, M.R.G.; NASCIMENTO, S.P.; SATO, E.T. Contribuição do PRONAF para o crescimento das economias locais: Uma análise para o Brasil e Regiões. Economia \& Região, Londrina (Pr), v.2, n.1, p.7-27, jan./jul. 2014.

SCHNEIDER, S.; FIALHO, M. A. V. Pobreza rural, desequilíbrios regionais e desenvolvimento agrário no Rio Grande do Sul. Teoria e Evidência Econômica, Passo Fundo-RS, v. 8, n. 15, p. 117-149, 2000.

SEN, A. Desigualdade Reexaminada. Rio de Janeiro, Ed. Record, 2001.

SILVA, J.G. A nova dinâmica da agricultura brasileira. 2 ed. rev. Campinas: Unicamp, 1998. $211 \mathrm{p}$.

SILVA, M.C.P.; BARROS, R.P. Pobreza multidimensional no Brasil. Anais da ANPEC Nacional 2006. Disponível em http.:// http://www.anpec.org.br/encontro2006/artigos/A06A141.pdf. Acessado em 13 de outubro de 2014.

SILVA JUNIOR, L.H. Pobreza na população nordestina: Uma análise das suas características durante os anos noventa. Revista do BNDES, Rio de Janeiro, v.13, n.26, p.275-290, 2006.

WORDBANK. Relatório sobre o desenvolvimento mundial de 2008: agricultura para o desenvolvimento. 2008. Disponível em:

http://siteresources. worldbank.org/INTWDRS/Resources/477365-1327599046334/8394679-

1327614067045/FINAL_WDR-OV-Portuguese-text_9.26.07.pdf 